Review

\title{
Kynurenines and Multiple Sclerosis: The Dialogue between the Immune System and the Central Nervous System
}

\author{
Cecilia Rajda ${ }^{1}$, Zsófia Majláth ${ }^{1}$, Dániel Pukoli ${ }^{1,2}$ and László Vécsei ${ }^{1,3, *}$ \\ 1 Department of Neurology, University of Szeged, Szeged H-6725, Hungary; \\ E-Mails: rajda.cecilia@med.u-szeged.hu (C.R.); majlathzsofia@gmail.com (Z.M.); \\ pukoli.daniel@med.u-szeged.hu (D.P.) \\ 2 Department of Neurology, Markhot Ferenc County Hospital, Eger H-3300, Hungary \\ 3 MTA-SZTE Neuroscience Research Group, Szeged H-6725, Hungary \\ * Author to whom correspondence should be addressed; E-Mail: vecsei.laszlo@med.u-szeged.hu; \\ Tel.: +36-62-545-351; Fax: +36-62-545-597.
}

Academic Editor: Christoph Kleinschnitz

Received: 15 June 2015 / Accepted: 23 July 2015 / Published: 6 August 2015

\begin{abstract}
Multiple sclerosis is an inflammatory disease of the central nervous system, in which axonal transection takes place in parallel with acute inflammation to various, individual extents. The importance of the kynurenine pathway in the physiological functions and pathological processes of the nervous system has been extensively investigated, but it has additionally been implicated as having a regulatory function in the immune system. Alterations in the kynurenine pathway have been described in both preclinical and clinical investigations of multiple sclerosis. These observations led to the identification of potential therapeutic targets in multiple sclerosis, such as synthetic tryptophan analogs, endogenous tryptophan metabolites (e.g., cinnabarinic acid), structural analogs (laquinimod, teriflunomid, leflunomid and tranilast), indoleamine-2,3-dioxygenase inhibitors (1MT and berberine) and kynurenine-3-monooxygenase inhibitors (nicotinylalanine and Ro 61-8048). The kynurenine pathway is a promising novel target via which to influence the immune system and to achieve neuroprotection, and further research is therefore needed with the aim of developing novel drugs for the treatment of multiple sclerosis and other autoimmune diseases.
\end{abstract}

Keywords: kynurenine pathway; multiple sclerosis; indoleamine-2,3-dioxygenase 


\section{Introduction}

Multiple sclerosis (MS) is a chronic disease with mainly inflammatory features in the beginning and later neurodegenerative processes take over. There is an overlap of the pathological processes during the time course. Patients are considered to be clinically active if they have two relapses in two years and may have gadolinium enchancing lesion(s) on brain magnetic resonance imaging (MRI). Chronic patients have no relapses, their disease course is slowly progressing from the beginning (primary progressive course) or slowly progressing after a relapsing-remitting course (secondary progressive). It became more and more evident that the acute plaques in the brain differ from the chronic plaques regarding their underlying pathomechanism.

In this inflammatory disease of the central nervous system (CNS) axonal transection takes place in parallel with acute inflammation. The extents of the two major pathomechanisms are individual. The demyelination appears to be extensive also in the gray matter and global neuron degeneration is also observed. The inflammation affects the energy metabolism of the axons through the mitochondria, and local oedema influences the local blood flow causing ischemic axonal degeneration. Toxic mediators lead to breakdown of the blood-brain barrier and damage to the myelin sheath and axons. Those mediators are tumor necrosis factor- $\alpha$ (TNF- $\alpha$ ), interferon- $\gamma$ (IFN- $\gamma)$, nitrogen-monoxid (NO) and reactive oxygen species.

The pathomechanism in MS is heterogeneous, but in a given individual the same pattern is present throughout the disease course. In the active inflammatory form, four subtypes have been described, which differ as regards the molecules taking part in the process: (1) T cell and macrophage-mediated; (2) B cell (antibody) and complement-mediated; (3) oligodendrocyte apoptosis-induced and (4) A primary oligodendrocyte apoptotic form.

In sequence of appearence, the most common form is 2, followed by 3, 1 and 4 [1].

The inflammation in MS may trigger a pathogenetic cascade of events leading to neurodegeneration, amplified by mechanisms related to brain aging and an accumulated disease burden. The factors causing this neurodegeneration include chronic oxidative injury, microglia activation, accumulation of mitochondrial damage in the axons and age-related iron accumulation in the brain. An altered mitochondrial function can cause neuronal death by the inbalance of ionic homeostasis and chronic cell stress [2]. The most characteristic lesion of MS is demyelination where the axons are partially preserved. However, in progressive MS brain atrophy is the most prominent pathological finding. In the course of relapsing-remitting MS (RRMS), actively demyelinating plaques associated with inflammation and blood-brain barrier injury are observed, but they are rare in the progressive course [2].

In chronic MS, the lesions shrink even in the presence of ongoing activity at the margins, contributing to brain atrophy. It is caused in part by the degeneration of the chronically demyelinated axons. Axonal degeneration begins in active MS lesions. Most axons survive the acute but not the chronic states of demyelination. In progressive MS, therefore, the degeneration of chronically demyelinated axons is a prominent feature, and is also a major cause of irreversible disability [2].

The blood-brain barrier damage observed with inflammation in the active white matter lesions in early MS differs in patients with progressive MS, in whom perivascular and parenchymal inflammation are seen, at least partly, in the absence of serum protein leakage from affected blood vessels. This means 
that such an inflammatory reaction is no longer reflected by contrast enhancement on MRI, and drugs that target this type of inflammation should be able to enter the CNS through an intact blood-brain barrier [2].

Another pathological feature of progressive MS is cortical demyelination, which is probably one of the causes of the cognitive disability suffered by the patients $[3,4]$. While early relapsing and remitting disease is associated with the appearance of focal plaques in the white matter, cortical demyelination, and diffuse alterations of the white and gray matter are present in the progressive stage [2].

\section{The Kynurenine Pathway}

The essential aminoacid tryptophan (Trp) can be metabolized in two main routes: the serotonin and the kynurenine pathways (KP) [5,6]. The serotonin pathway is better known, though more than $95 \%$ of the Trp is transformed through the KP [7]. The first and rate-limiting step of the KP, the enzymatic transformation of Trp into L-kynurenine (L-KYN), can be catalyzed by either tryptophan-2,3-dioxygenase (TDO) or indoleamine-2,3-dioxygenase (IDO). TDO is predominantly located in the liver cells, but in smaller amounts it can be found in the brain as well. IDO is present in numerous different cell types such as the microglia, neurones or astrocytes. At the level of L-KYN, the KP divides into three branches: it can be metabolized into kynurenic acid (KYNA), or it can produce anthranilic acid or it will be degraded in a sequence of enzymatic steps responsible for the formation of neurotoxic kynurenines. KYNA is produced by kynurenine-aminotransferases (KATs). The four known KAT isoforms have slightly different biochemical properties; KAT-II is mainly responsible for KYNA synthesis in the human brain. KYNA is the only known endogenous antagonist of ionotropic glutamate receptors, and it therefore has a significant neuroprotective property [8]. It is a competitive antagonist of $N$-methyl-D-aspartate (NMDA) receptors $[9,10]$. Interestingly, it has a dose-dependent dual effect on the $\alpha$-amino-3-hydroxy5-methyl-4-isoxazolepropionic acid (AMPA) receptors: in lower concentrations it evokes facilitation, while in higher amounts it has an inhibitory effect $[11,12]$. Another important effect of KYNA is the inhibiton of presynaptic $\alpha 7$ nicotinic acetylcholine receptors thereby regulating presynaptic glutamate release $[13,14]$. As even low concentrations of KYNA are able to inhibit glutamate release, this effect contributes significantly to its neuroprotective effect besides NMDA antagonism.

Another branch of the KP produces several neuroactive kynurenines which are mainly neurotoxic. 3-hydroxy-kynurenine (3HK) is formed from L-KYN through the action of kynurenine-3-monooxygenase (KMO). The third branch of the KP is the formation of anthranilic acid from L-KYN by the action of kynureninase. Anthranilic acid and $3 \mathrm{HK}$ are both converted into 3-hydroxy-anthranilic acid, which is degraded further and yields quinolinic acid (QUIN). 3HK and QUIN are both neurotoxic molecules, which in higher conentrations may result in neuronal damage. QUIN is a potent agonist of NMDA receptors, which may thereby induce glutamatergic excitotoxicity [15]. QUIN may also induce lipid peroxidation and oxidative stress [16]. 3HK and 3-hydroxy-anthranilic acid contribute to free radical production and therefore strengthen the neurotoxic effects of QUIN. The enzymatic machinery is differently distributed in the different cell types: the astrocytes harbor mainly KATs and hardly any $\mathrm{KMO}$, while microglial cells have predominantly $\mathrm{KMO}$ and therefore produce mainly the neurotoxic kynurenines [17,18]. 


\section{The Role of the KP in Immunoregulation}

The importance of the KP in the physiological functions and pathological processes of the nervous system has been extensively investigated, and it has recently been implicated as having a regulatory function in the immune system too [19]. In this relation, IDO is of outstanding importance, and may take part in the immunoregulation through Trp depletion and the production of kynurenines. IDO is present in various immune cells, including monocytes, macrophages and microglia [20]. IDO can be induced by interferons and LPS too, but IFN- $\gamma$ is considered to be the main activator. The activation of IDO results in an elevation of the levels of KYNA and other kynurenines, and it is considered to be an important regulator of immune activation as it counteracts the proliferation of reactive lymphocytes. This is partly a result of Trp depletion, while on the other hand, QUIN and 3-hydroxyanthranilic acid result in the selective apoptosis of TH1 cells [21]. This function is considered to play a significant role in the regulation of the immune response as a negative feedback loop and in the development of immune tolerance [22,23]. Importantly, IDO-mediated T-cell inhibition prefers Th1 cells, though, it may additionally counteract $\mathrm{Th} 2$ cells through the activation of regulatory T-cells [20].

\section{The Role of the KP in the Pathomechanism of MS}

\subsection{Preclinical Results}

Experimental autoimmune encephalitis (EAE) is a T-cell-mediated autoimmune animal model for MS that is histologically similar to human MS [24,25]. The EAE is until now the best animal model representing the relapsing-remitting form of the disease, however there are known shortcomes, e.g., the progressive phase is not modelled, etc. Kwidzinski et al. [26] demonstrated the importance of the immune modulating effect of IDO-1 in EAE. The inhibition of the enzyme activity significantly decreases the neuroinflammatory process, resulting in a decrease of the exacerbation of the disease. In the spinal cord and brainstem of rats with EAE, KMO immunoreactivity has been found in the cytoplasmic granules. Another neurotoxic KP metabolite, 3-HK, has been found to be increased in the spinal cords of rats with EAE [27]. Flanagan et al. [28] measured a selective QUIN level increase in the spinal cords of EAE rats. Both EAE models have been induced by the same methods, resulting in an acute clinical course. Cammer et al. [29-31] observed that QUIN in pathologic concentrations ( 0.1 and $1 \mathrm{mM})$ causes oligodendrocyte apoptosis. Neuronal, astroglial, and oligodendroglial cell death has been described on chronic QUIN exposure. Pierozan et al. [32] revealed that QUIN changes the structures of various proteins (tau, neurofilaments, etc.), resulting in neuron degeneration.

\subsection{Clinical Results}

The evidence of subjects with MS found by Monaco et al. [33-35] that the Trp levels are lower in the serum and CSF corresponds with more recent results regarding depressed TRP levels in the serum and CSF of MS patients, proving the activation of KP in MS. An enhanced level of L-KYN was found in IFN- $\beta$ treated MS patients relative to untreated RRMS patients [36]. Anderson et al. [37] identified a possible new role for QUIN, since they observed abnormal tau phosphorylation in progressive MS. Moreover, IDO-1 plays a very important role in regulating the immune response. During MS progression, it is very likely that the levels of pro-inflammatory cytokines such as IFN- $\gamma$ and TNF- $\alpha$ 
increase in MS patients, thereby leading to IDO-1 and KP activation [38]. A number of research groups have found that many proinflammatory cytokines can activate IDO-1 [39-41]. Evidence has been presented that IFN- $\beta$ can activate both pathways of the kynurenine cascade in the plasma of MS patients, and that IFN- $\beta$ also affects IDO-1 (though to a lesser extent), which in turn decreases QUIN production [42]. In 2005, Hartai et al. [43] reported that serum levels of the KAT I and KAT II were significantly higher in the red blood cells of MS patients than in controls. Furthermore, the concentration of KYNA was found to be elevated in the plasma of MS patients; the same group also described the possible neuroprotective effect of KYNA in MS [43]. Elevated KYNA levels were described in the CSF of MS patiens [44]. The opposite was found in postmortem MS brain sections, with decreases in the concentrations of both enzymes responsible for KYNA production [45]. A low KYNA serum level was measured in the CSF of MS patiens in the remission [46]. Interestingly, elevated levels were found in acute relapse [47]. These controversial results may have originated from the disease groups (relapsing versus progressive) not being homogeneous at the time of sampling among the cited studies, e.g., kynurenines have been measured in different phases of the disease [47]. These findings point to the possible preventing role of KYNA in the acute phase of MS. The lower KYNA levels seen in the progressive phase of the disease shift the KP to neurotoxicity. Alterations of the KP have been revealed in all phases of the disease. To summarize the above results, KYNA is involved in possible neurotoxic processes as a protective agent, underlining its importance in neurodegenerative mechanisms (Figure 1, Table 1).

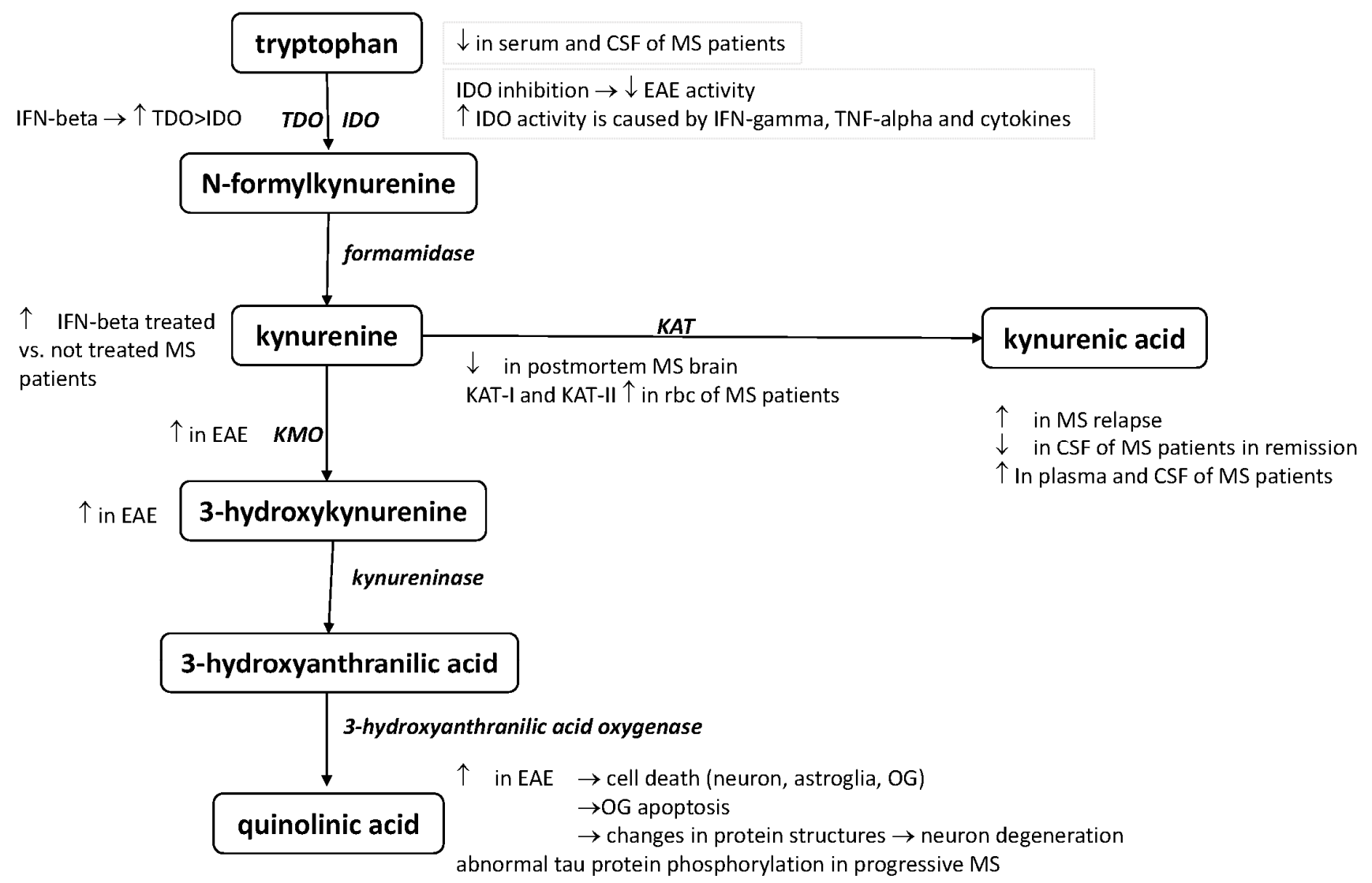

Figure 1. Alterations of the KP in EAE and MS. Alterations in the kynurenine pathway in EAE and MS - this schematic picture summarizes the alterations of the different kynurenine metabolites and enzymes in the animal model and human disease based on the presently published data. For list of abbreviations see page 9. 
Table 1. The role of kynurenine pathway in MS and its experimental animal model-findings in connection with the disease.

\begin{tabular}{|c|c|}
\hline $\begin{array}{l}\text { Alterations of the KP Metabolism } \\
\end{array}$ & References \\
\hline Elevated serum and CSF TRP levels in MS patients with acute relapse & [33] \\
\hline Depressed serum and CSF TRP levels in MS & {$[34,35]$} \\
\hline $\begin{array}{c}\text { KAT I and KAT II serum levels were significantly higher in red blood } \\
\text { cells of MS patients }\end{array}$ & {$[43]$} \\
\hline $\begin{array}{c}\text { Decrease the concentrations of KAT I and KAT II enzymes in } \\
\text { postmortem MS brain sections }\end{array}$ & {$[45]$} \\
\hline KYNA concentrations elevated in the plasma of MS patients & [43] \\
\hline Elevated KYNA levels in the cerebrospinal fluid of MS patients & {$[44]$} \\
\hline Low KYNA serum level in the CSF of MS patients in remission & [46] \\
\hline Elevated KYNA level in the CSF with acute relapse & [47] \\
\hline 3-HK is increased in EAE rats & [27] \\
\hline $\begin{array}{l}\text { KMO enzyme immunoreactivity has been found in cytoplasmic granules } \\
\text { in the spinal cord and brainstem of rats with EAE }\end{array}$ & {$[27]$} \\
\hline QUIN level increased in the spinal cords of EAE rats & [28] \\
\hline
\end{tabular}

\section{Possible Therapeutic Targets Related to the KP}

The treatments currently available for MS are all anti-inflammatory as concerns the mechanism of their effects, reducing the number and duration of attacks. At the present time, no neuroprotective molecules that would facilitate remyelination are available. An NMDA receptor antagonist was reported to modify the course of EAE and to prevent blood-brain barrier breakdown [48]. The NMDA antagonist KYNA and its pharmacological derivatives, together with KP enzyme inhibitors, might be promising new drugs that could fill the therapeutic gap (Figure 2). KYNA, a neuroprotective TRP metabolite, is itself not able to cross the blood-brain barrier; passage is possible by the ester form or by KYNA analogs [49]. One possible mode of beneficial therapeutic intervention is to shift the KP metabolism toward the formation of protective agents. This metabolic shift can be achieved by specific inhibitors of KMO, kynureninase and 3-hydroxi-anthranilic acid dioxygenase. A considerable number of possible pharmaceuticals have been developed with the aim of inhibiting these enzymes [50-52]. The systematic use of Ro 61-8048 as a selective KMO inhibitor led to accumulation of the neuroprotective KYNA and to decreases in the elevation of both $3 \mathrm{HK}$ and QUIN in the rat spinal cord [27]. An orally active synthetic Trp metabolite ( $N$-[3,4-dimethoxycinnamoyl]-anthranilic acid (3,4-DAA), also known as Tranilas) has been examined in both in vitro and in vivo experiments (in microglial cells and in the RR version of EAE). Platten et al. [53] showed that this synthetic Trp metabolite decreased the proliferation of myelin-specific T cells and inhibited the proinflammatory cytokines produced by TH1 cells. The animals treated with 3,4-DAA suffered fewer and milder relapses, and there were decreases in the number of inflammatory nodes in the brains and spinal cords of the treated mice, verifying the immunosuppressive effect of this molecule, and indicating its possible use in TH1-mediated autoimmune diseases, e.g., MS. Laquinimod, a quinoline carboxamide, displays structural similarities with the Trp metabolites 3-HAA, 
3-HKA and KYNA, and it might cross the blood-brain barrier [54-56]. One study revealed the role of laquinimod in immunoregulation, as it proved to decrease the antigen presentation and T-cell chemotaxis in APCs, and to shift the immune response from Th1 to Th2 and to affect Treg cells [57-61]. Similarly to glatiramer acetate, laquinimod treatment promoted the development of regulatory monocytes, revealing an increased secretion of IL-10. This made it capable of inhibiting the secretion of the proinflammatory cytokine IFN- $\gamma$ [62]. Besides shifting the cytokine responses toward Th2, it prevented the proliferation of autoreactive T cells in the CNS. In a phase 3 study, laquinimod decreased the progression of the damage, and also the annual relapse rate to a lesser extent [63].

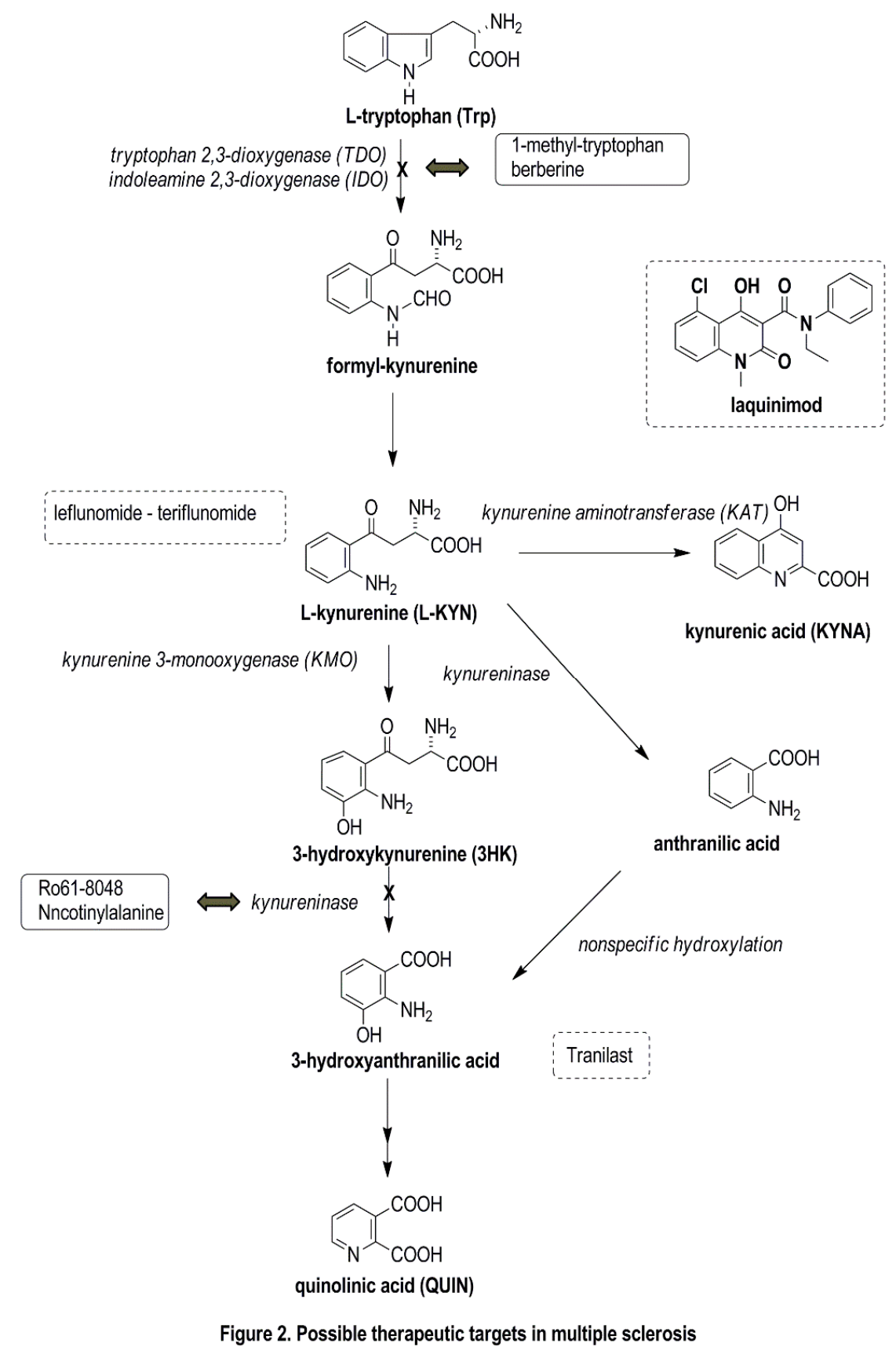

Figure 2. Possible therapeutic targets in MS. Possible therapeutic targets in the kynurenine pathway - the figure displays the future drug candidates which are influencing the kynurenine pathway (molecules indicated in the frames) or have structural similarities (represented in dashed line boxes) with kynurenine metabolites. 
Sundaram et al. [64] inhibited the production of IDO-1 in a BV2 microglial cell culture, which in turn decreased QUIN production. IDO-1 inhibitors (1-MT and berberine) at $4 \mu \mathrm{M}$ concentration significantly affected the QUIN levels and fully eliminated the oligodendrocyte apoptosis. The use of KP inhibitors could be a new strategy in the treatment of MS.

Cinnabarinic acid is an endogenous tryptophan metabolite of the KP, and a partial agonist of the type-4 metabotropic glutamate receptor (mGluR4), which proved to be protective in EAE [65]. Fallarino et al. [66] observed Th17 cell differentiation in the peripheral dendritic cells of mGluR4 knockout EAE rodents after initiation of the immune response. The expression of mGluR4 was found in wild-type EAE mice. After cinnabarinic acid administration, the expression of mGluR4 activation led to $\mathrm{T}$ cell differentiation in favor of the Th17 cells, which are responsible for immune tolerance and most probably protection against EAE. When Fazio et al. [67] treated mGlu4 knockout mice with cinnabarinic acid, the immune response was shifted toward $\mathrm{T}$ reg cell production. The latter offers a new therapeutic option in MS, by activating the KP pathway, changing the immunotolerance, and leading to a decrease in neuroinflammation.

An increase in interleukin-18 level, which activates the induction of IDO and QUIN, may partially mediate seizure activity in MS by elevating the IFN- $\gamma$ level. Through this route, antiepileptic drugs might modulate wider MS symptomatology, interfering with melatonin and vitamin D3 [68].

\section{Conclusions}

Several endogenous neuroprotective mechanisms have recently been identified in MS, involving protective autoimmunity, direct low molecular weight antioxidants, indirect antioxidants inducing cytoprotective proteins, kynurenines, ischemic preconditioning, an integrated cell response, cannabinoids and the complement system. The result depends on the microenvironmental system [69]. The KP is a promising novel target through which to influence the immune responses and to achieve neuroprotection. Further research is therefore needed with the aim of developing novel therapeutics in MS and other autoimmune diseases.

\section{Acknowledgments}

This work was supported by the project TÁMOP-4.2.6.3.1, by the Hungarian Brain Research Programme (NAP, Grant No. KTIA_13_NAP-A-III/9 and KTIA_13_NAP-A-II/17) and by the MTA-SZTE Neuroscience Research Group of the Hungarian Academy of Sciences and the University of Szeged.

Thanks are due to David Durham for the linguistic corrections of the manuscript.

\section{Conflicts of Interest}

The authors declare no conflict of interest. 


\section{List of Abbreviations}

\begin{tabular}{|c|c|}
\hline 3HK & 3-hydroxi-kynurenine \\
\hline AMPA & $\alpha$-amino-3-hydroxy-5-methyl-4-isoxazolepropionic acid \\
\hline CSF & cerebrospinal fluid \\
\hline EAE & experimental autoimmune encephalomyelitis \\
\hline IDO & indoleamine-2,3-dioxygenase \\
\hline IFN- $\beta$ & interferon-beta \\
\hline IFN- $\gamma$ & kynurenine-aminotransferase \\
\hline KAT & kynurenine-3-monooxygenase \\
\hline KMO & kynurenine pathway \\
\hline KP & kynurenic acid \\
\hline KYNA & multiple sclerosis \\
\hline L-KYN & magnetic resonance imaging \\
\hline MS & $N$-methyl-D-aspartate \\
\hline MRI & nitrogen-monoxid \\
\hline NMDA & oligodendrocyte \\
\hline NO & quinolinic acid \\
\hline OG & relapsing-remitting multiple sclerosis \\
\hline QUIN & tryptophane-2,3-dioxygenase \\
\hline RRMS & tumor necrosis factor-alpha \\
\hline TDO & tryptophan \\
\hline TNF- $\alpha$ & \\
\hline Trp & \\
\hline &
\end{tabular}

\section{References}

1. Lassmann, H.; Bruck, W.; Lucchinetti, C.F. The immunopathology of multiple sclerosis: An overview. Brain Pathol. 2007, 17, 210-218.

2. Mahad, D.H.; Trapp, B.D.; Lassmann, H. Pathological mechanisms in progressive multiple sclerosis. Lancet Neurol. 2015, 14, 183-193.

3. Nielsen, A.S.; Kinkel, R.P.; Madigan, N.; Tinelli, E.; Benner, T.; Mainero, C. Contribution of cortical lesion subtypes at 7T MRI to physical and cognitive performance in MS. Neurology 2013, 81, 641-649.

4. Peterson, J.W.; Bo, L.; Mork, S.; Chang, A.; Trapp, B.D. Transected neurites, apoptotic neurons, and reduced inflammation in cortical multiple sclerosis lesions. Ann. Neurol. 2001, 50, 389-400.

5. Schwarcz, R.; Bruno, J.P.; Muchowski, P.J.; Wu, H.Q. Kynurenines in the mammalian brain: When physiology meets pathology. Nat. Rev. Neurosci. 2012, 13, 465-477.

6. Vecsei, L. Kynurenines in the Brain: From Experiments to Clinics; Nova Science Publishers: Hauppauge, NY, USA, 2005.

7. Leklem, J.E. Quantitative aspects of tryptophan metabolism in humans and other species: A review. Am. J. Clin. Nutr. 1971, 24, 659-672.

8. Han, Q.; Cai, T.; Tagle, D.A.; Li, J. Structure, expression, and function of kynurenine aminotransferases in human and rodent brains. Cell Mol. Life Sci. 2010, 67, 353-368. 
9. Kessler, M.; Terramani, T.; Lynch, G.; Baudry, M. A glycine site associated with $N$-methyl-D-aspartic acid receptors: Characterization and identification of a new class of antagonists. J. Neurochem. 1989, 52, 1319-1328.

10. Stone, T.W. Neuropharmacology of quinolinic and kynurenic acids. Pharmacol. Rev. 1993, 45, 309-379.

11. Prescott, C.; Weeks, A.M.; Staley, K.J.; Partin, K.M. Kynurenic acid has a dual action on AMPA receptor responses. Neurosci. Lett. 2006, 402, 108-112.

12. Rozsa, E.; Robotka, H.; Vecsei, L.; Toldi, J. The Janus-face kynurenic acid. J. Neural. Transm. 2008, 115, 1087-1091.

13. Hilmas, C.; Pereira, E.F.; Alkondon, M.; Rassoulpour, A.; Schwarcz, R.; Albuquerque, E.X. The brain metabolite kynurenic acid inhibits $\alpha 7$ nicotinic receptor activity and increases non- $\alpha 7$ nicotinic receptor expression: Physiopathological implications. J. Neurosci. 2001, 21, 7463-7473.

14. Marchi, M.; Risso, F.; Viola, C.; Cavazzani, P.; Raiteri, M. Direct evidence that release-stimulating $\alpha 7$ nicotinic cholinergic receptors are localized on human and rat brain glutamatergic axon terminals. J. Neurochem. 2002, 80, 1071-1078.

15. Stone, T.W.; Perkins, M.N. Quinolinic acid: A potent endogenous excitant at amino acid receptors in CNS. Eur. J. Pharmacol. 1981, 72, 411-412.

16. Perez-De La Cruz, V.; Carrillo-Mora, P.; Santamaria, A. Quinolinic acid, an endogenous molecule combining excitotoxicity, oxidative stress and other toxic mechanisms. Int. J. Tryptophan Res. 2012, 5, 1-8.

17. Guillemin, G.J.; Cullen, K.M.; Lim, C.K.; Smythe, G.A.; Garner, B.; Kapoor, V.; Takikawa, O.; Brew, B.J. Characterization of the kynurenine pathway in human neurons. J. Neurosci. 2007, 27, 12884-12892.

18. Guillemin, G.J.; Kerr, S.J.; Smythe, G.A.; Smith, D.G.; Kapoor, V.; Armati, P.J.; Croitoru, J.; Brew, B.J. Kynurenine pathway metabolism in human astrocytes: A paradox for neuronal protection. J. Neurochem. 2001, 78, 842-853.

19. Vecsei, L.; Szalardy, L.; Fulop, F.; Toldi, J. Kynurenines in the CNS: Recent advances and new questions. Nat. Rev. Drug Discov. 2013, 12, 64-82.

20. Mandi, Y.; Vecsei, L. The kynurenine system and immunoregulation. J. Neural. Transm. 2012, 119, 197-209.

21. Fallarino, F.; Grohmann, U.; Vacca, C.; Bianchi, R.; Orabona, C.; Spreca, A.; Fioretti, M.C.; Puccetti, P. T cell apoptosis by tryptophan catabolism. Cell Death Differ. 2002, 9, 1069-1077.

22. Belladonna, M.L.; Puccetti, P.; Orabona, C.; Fallarino, F.; Vacca, C.; Volpi, C.; Gizzi, S.; Pallotta, M.T.; Fioretti, M.C.; Grohmann, U. Immunosuppression via tryptophan catabolism: The role of kynurenine pathway enzymes. Transplantation 2007, 84, S17-S20.

23. Gonzalez, A.; Varo, N.; Alegre, E.; Diaz, A.; Melero, I. Immunosuppression routed via the kynurenine pathway: A biochemical and pathophysiologic approach. Adv. Clin. Chem. 2008, 45, 155-197.

24. Paterson, P.Y. Autoimmune diseases of myelin. Prog. Clin. Biol. Res. 1980, 49, 19-36.

25. Gold, R.; Linington, C.; Lassmann, H. Understanding pathogenesis and therapy of multiple sclerosis via animal models: 70 years of merits and culprits in experimental autoimmune encephalomyelitis research. Brain 2006, 129, 1953-1971. 
26. Kwidzinski, E.; Bunse, J.; Aktas, O.; Richter, D.; Mutlu, L.; Zipp, F.; Nitsch, R.; Bechmann, I. Indolamine 2,3-dioxygenase is expressed in the CNS and down-regulates autoimmune inflammation. FASEB J. 2005, 19, 1347-1349.

27. Chiarugi, A.; Cozzi, A.; Ballerini, C.; Massacesi, L.; Moroni, F. Kynurenine 3-mono-oxygenase activity and neurotoxic kynurenine metabolites increase in the spinal cord of rats with experimental allergic encephalomyelitis. Neuroscience 2001, 102, 687-695.

28. Flanagan, E.M.; Erickson, J.B.; Viveros, O.H.; Chang, S.Y.; Reinhard, J.F., Jr. Neurotoxin quinolinic acid is selectively elevated in spinal cords of rats with experimental allergic encephalomyelitis. J. Neurochem. 1995, 64, 1192-1196.

29. Cammer, W. Oligodendrocyte killing by quinolinic acid in vitro. Brain Res. 2001, 896, 157-160.

30. Guillemin, G.J.; Wang, L.; Brew, B.J. Quinolinic acid selectively induces apoptosis of human astrocytes: Potential role in AIDS dementia complex. J. Neuroinflamm. 2005, 2, 16.

31. Kerr, S.J.; Armati, P.J.; Guillemin, G.J.; Brew, B.J. Chronic exposure of human neurons to quinolinic acid results in neuronal changes consistent with aids dementia complex. AIDS 1998, 12, 355-363.

32. Pierozan, P.; Zamoner, A.; Soska, A.K.; Silvestrin, R.B.; Loureiro, S.O.; Heimfarth, L.; Mello e Souza, T.; Wajner, M.; Pessoa-Pureur, R. Acute intrastriatal administration of quinolinic acid provokes hyperphosphorylation of cytoskeletal intermediate filament proteins in astrocytes and neurons of rats. Exp. Neurol. 2010, 224, 188-196.

33. Monaco, F.; Fumero, S.; Mondino, A.; Mutani, R. Plasma and cerebrospinal fluid tryptophan in multiple sclerosis and degenerative diseases. J Neurol. Neurosurg. Psychiatry 1979, 42, 640-641.

34. Rudzite, V.; Berzinsh, J.; Grivane, I.; Fuchs, D.; Baier-Bitterlich, G.; Wachter, H. Serum tryptophan, kynurenine, and neopterin in patients with Guillain-Barre-syndrome (GBS) and multiple sclerosis (MS). Adv. Exp. Med. Biol. 1996, 398, 183-187.

35. Sandyk, R. Tryptophan availability and the susceptibility to stress in multiple sclerosis: A hypothesis. Int. J. Neurosci. 1996, 86, 47-53.

36. Sadowska-Bartosz, I.; Adamczyk-Sowa, M.; Gajewska, A.; Bartosz, G. Oxidative modification of blood serum proteins in multiple sclerosis after interferon or mitoxantrone treatment. J. Neuroimmunol. 2014, 266, 67-74.

37. Anderson, J.M.; Hampton, D.W.; Patani, R.; Pryce, G.; Crowther, R.A.; Reynolds, R.; Franklin, R.J.; Giovannoni, G.; Compston, D.A.; Baker, D.; et al.. Abnormally phosphorylated tau is associated with neuronal and axonal loss in experimental autoimmune encephalomyelitis and multiple sclerosis. Brain 2008, 131, 1736-1748.

38. Beck, J.; Rondot, P.; Catinot, L.; Falcoff, E.; Kirchner, H.; Wietzerbin, J. Increased production of interferon $\gamma$ and tumor necrosis factor precedes clinical manifestation in multiple sclerosis: Do cytokines trigger off exacerbations? Acta Neurol. Scand. 1988, 78, 318-323.

39. Pemberton, L.A.; Kerr, S.J.; Smythe, G.; Brew, B.J. Quinolinic acid production by macrophages stimulated with IFN- $\gamma$, TNF- $\alpha$, and IFN- $\alpha$. J. Interferon Cytokine Res. 1997, 17, 589-595.

40. Smith, D.G.; Guillemin, G.J.; Pemberton, L.; Kerr, S.; Nath, A.; Smythe, G.A.; Brew, B.J. Quinolinic acid is produced by macrophages stimulated by platelet activating factor, nef and tat. J. Neurovirol. 2001, 7, 56-60.

41. Guillemin, G.J.; Smith, D.G.; Smythe, G.A.; Armati, P.J.; Brew, B.J. Expression of the kynurenine pathway enzymes in human microglia and macrophages. Adv. Exp. Med. Biol. 2003, 527, 105-112. 
42. Guillemin, G.J.; Kerr, S.J.; Pemberton, L.A.; Smith, D.G.; Smythe, G.A.; Armati, P.J.; Brew, B.J. IFN- $\beta 1 \mathrm{~b}$ induces kynurenine pathway metabolism in human macrophages: Potential implications for multiple sclerosis treatment. J. Interferon Cytokine Res. 2001, 21, 1097-1101.

43. Hartai, Z.; Klivenyi, P.; Janaky, T.; Penke, B.; Dux, L.; Vecsei, L. Kynurenine metabolism in multiple sclerosis. Acta Neurol. Scand. 2005, 112, 93-96.

44. Kepplinger, B.; Baran, H.; Kainz, A.; Ferraz-Leite, H.; Newcombe, J.; Kalina, P. Age-related increase of kynurenic acid in human cerebrospinal fluid-IgG and $\beta 2$-microglobulin changes. Neuro Signals 2005, 14, 126-135.

45. Lim, C.K.; Brew, B.J.; Sundaram, G.; Guillemin, G.J. Understanding the roles of the kynurenine pathway in multiple sclerosis progression. Int. J. Tryptophan Res. 2010, 3, 157-167.

46. Rejdak, K.; Bartosik-Psujek, H.; Dobosz, B.; Kocki, T.; Grieb, P.; Giovannoni, G.; Turski, W.A.; Stelmasiak, Z. Decreased level of kynurenic acid in cerebrospinal fluid of relapsing-onset multiple sclerosis patients. Neurosci. Lett. 2002, 331, 63-65.

47. Rejdak, K.; Petzold, A.; Kocki, T.; Kurzepa, J.; Grieb, P.; Turski, W.A.; Stelmasiak, Z. Astrocytic activation in relation to inflammatory markers during clinical exacerbation of relapsing-remitting multiple sclerosis. J. Neural. Transm. 2007, 114, 1011-1015.

48. Paul, C.; Bolton, C. Modulation of blood-brain barrier dysfunction and neurological deficits during acute experimental allergic encephalomyelitis by the $N$-methyl-D-aspartate receptor antagonist memantine. J. Pharmacol. Exp. Ther. 2002, 302, 50-57.

49. Stone, T.W. Kynurenic acid antagonists and kynurenine pathway inhibitors. Exp. Opin. Investig. Drugs 2001, 10, 633-645.

50. Chiarugi, A.; Carpenedo, R.; Molina, M.T.; Mattoli, L.; Pellicciari, R.; Moroni, F. Comparison of the neurochemical and behavioral effects resulting from the inhibition of kynurenine hydroxylase and/or kynureninase. J. Neurochem. 1995, 65, 1176-1183.

51. Colabroy, K.L.; Zhai, H.; Li, T.; Ge, Y.; Zhang, Y.; Liu, A.; Ealick, S.E.; McLafferty, F.W.; Begley, T.P. The mechanism of inactivation of 3-hydroxyanthranilate-3,4-dioxygenase by 4-chloro-3-hydroxyanthranilate. Biochemistry 2005, 44, 7623-7631.

52. Walsh, H.A.; O'Shea, K.C.; Botting, N.P. Comparative inhibition by substrate analogues 3-methoxy- and 3-hydroxydesaminokynurenine and an improved 3 step purification of recombinant human kynureninase. BMC Biochem. 2003, 4, 13.

53. Platten, M.; Ho, P.P.; Youssef, S.; Fontoura, P.; Garren, H.; Hur, E.M.; Gupta, R.; Lee, L.Y.; Kidd, B.A.; Robinson, W.H.; et al. Treatment of autoimmune neuroinflammation with a synthetic tryptophan metabolite. Science 2005, 310, 850-855.

54. Bruck, W.; Wegner, C. Insight into the mechanism of laquinimod action. J. Neurol. Sci. 2011, 306, 173-179.

55. Fernandez, O. Oral laquinimod treatment in multiple sclerosis. Neurologia 2011, 26, 111-117.

56. Polman, C.; Barkhof, F.; Sandberg-Wollheim, M.; Linde, A.; Nordle, O.; Nederman, T. Treatment with laquinimod reduces development of active MRI lesions in relapsing MS. Neurology 2005, 64, 987-991.

57. Gurevich, M.; Gritzman, T.; Orbach, R.; Tuller, T.; Feldman, A.; Achiron, A. Laquinimod suppress antigen presentation in relapsing-remitting multiple sclerosis: In-vitro high-throughput gene expression study. J. Neuroimmunol. 2010, 221, 87-94. 
58. Zou, L.P.; Abbas, N.; Volkmann, I.; Nennesmo, I.; Levi, M.; Wahren, B.; Winblad, B.; Hedlund, G.; Zhu, J. Suppression of experimental autoimmune neuritis by ABR-215062 is associated with altered Th1/Th2 balance and inhibited migration of inflammatory cells into the peripheral nerve tissue. Neuropharmacology 2002, 42, 731-739.

59. Wegner, C.; Stadelmann, C.; Pfortner, R.; Raymond, E.; Feigelson, S.; Alon, R.; Timan, B.; Hayardeny, L.; Bruck, W. Laquinimod interferes with migratory capacity of T cells and reduces IL-17 levels, inflammatory demyelination and acute axonal damage in mice with experimental autoimmune encephalomyelitis. J. Neuroimmunol. 2010, 227, 133-143.

60. Aharoni, R.; Saada, R.; Eilam, R.; Hayardeny, L.; Sela, M.; Arnon, R. Oral treatment with laquinimod augments regulatory $\mathrm{T}$-cells and brain-derived neurotrophic factor expression and reduces injury in the CNS of mice with experimental autoimmune encephalomyelitis. J. Neuroimmunol. 2012, 251, 14-24.

61. Schulze-Topphoff, U.; Shetty, A.; Varrin-Doyer, M.; Molnarfi, N.; Sagan, S.A.; Sobel, R.A.; Nelson, P.A.; Zamvil, S.S. Laquinimod, a quinoline-3-carboxamide, induces type II myeloid cells that modulate central nervous system autoimmunity. PLoS ONE 2012, 7, e33797.

62. Weber, M.S.; Prod'homme, T.; Youssef, S.; Dunn, S.E.; Rundle, C.D.; Lee, L.; Patarroyo, J.C.; Stuve, O.; Sobel, R.A.; Steinman, L.; et al. Type II monocytes modulate T cell-mediated central nervous system autoimmune disease. Nat. Med. 2007, 13, 935-943.

63. Comi, G.; Jeffery, D.; Kappos, L.; Montalban, X.; Boyko, A.; Rocca, M.A.; Filippi, M. Placebo-controlled trial of oral laquinimod for multiple sclerosis. N. Eng. J. Med. 2012, 366, 1000-1009.

64. Sundaram, G.; Brew, B.J.; Jones, S.P.; Adams, S.; Lim, C.K.; Guillemin, G.J. Quinolinic acid toxicity on oligodendroglial cells: Relevance for multiple sclerosis and therapeutic strategies. J. Neuroinflamm. 2014, 11, 1-11.

65. Fazio, F.; Lionetto, L.; Molinaro, G.; Bertrand, H.O.; Acher, F.; Ngomba, R.T.; Notartomaso, S.; Curini, M.; Rosati, O.; Scarselli, P.; et al. Cinnabarinic acid, an endogenous metabolite of the kynurenine pathway, activates type 4 metabotropic glutamate receptors. Mol. Pharmacol. 2012, 81, 643-656.

66. Fallarino, F.; Volpi, C.; Fazio, F.; Notartomaso, S.; Vacca, C.; Busceti, C.; Bicciato, S.; Battaglia, G.; Bruno, V.; Puccetti, P.; et al. Metabotropic glutamate receptor-4 modulates adaptive immunity and restrains neuroinflammation. Nat. Med. 2010, 16, 897-902.

67. Fazio, F.; Zappulla, C.; Notartomaso, S.; Busceti, C.; Bessede, A.; Scarselli, P.; Vacca, C.; Gargaro, M.; Volpi, C.; Allegrucci, M.; et al. Cinnabarinic acid, an endogenous agonist of type-4 metabotropic glutamate receptor, suppresses experimental autoimmune encephalomyelitis in mice. Neuropharmacology 2014, 81, 237-243.

68. Anderson, G.; Rodriguez, M. Multiple sclerosis, seizures, and antiepileptics: Role of IL-18, IDO, and melatonin. Eur. J. Neurol. 2011, 18, 680-685.

69. Gonsette, R.E. Endogenous neuroprotection in multiple sclerosis. Acta Neurol. Belg. 2010, 110, $26-35$.

(C) 2015 by the authors; licensee MDPI, Basel, Switzerland. This article is an open access article distributed under the terms and conditions of the Creative Commons Attribution license (http://creativecommons.org/licenses/by/4.0/). 